\title{
A UNIQUENESS THEOREM FOR A CLASSICAL NONLINEAR SHALLOW SHELL MODEL
}

\author{
J. Cagnol, ${ }^{1}$ C.G. Lebiedzik, ${ }^{2}$ and R.J. Marchand ${ }^{3}$ \\ ${ }^{1}$ Pôle Universitaire Léonard de Vinci, ESILV, DER CS 92916 Paris La Défense Cedex, France, \\ John.Cagnol@devinci.fr * ${ }^{2}$ Wayne State University, 656 W. Kirby, Room 1150, Detroit, MI \\ 48202,USA, kate@math.wayne.edu ${ }^{\dagger},{ }^{3}$ Slippery Rock University, Slippery Rock, PA 16057, \\ USA, richard.marchand@sru.edu
}

\begin{abstract}
The main goal of this paper is to establish the uniqueness of solutions of finite energy for a classical dynamic nonlinear thin shallow shell model with clamped boundary conditions. The static representation of the model is an extension of a Koiter shallow shell model. Until now, this has been an open problem in the literature. The primary difficulty is due to a lack of regularity in the nonlinear terms. Indeed the nonlinear terms are not locally Lipshitz with respect to the energy norm. The proof of the theorem relies on sharp PDE estimates that are used to prove uniqueness in a lower topology than the space of finite energy.
\end{abstract}

keywords: Nonlinear shells, weak solutions, uniqueness

\section{Introduction}

The model considered governs the vibrations of a thin shallow shell structure. The importance of the model stems from a variety of engineering applications such as helicopter rotor blades, propellors, acoustic chambers (for noise suppression) with curved walls such as aircraft cabins, aircraft control surfaces, curved surfaces on modern turbo-jet engines, etc. The primary goals in applications are often to achieve uniform stability or control of the vibrations as described, for example, in [9]. These control theoretic applications coupled with necessary numerical methods for implementation require a thorough knowledge of existence and uniqueness of finite energy (weak) solutions. In the next subsection we will describe classical shell theory notation and present the variational form of the model followed by a brief review of related literature. An overview of the primary results and relevant proofs will comprise the remaining two sections.

${ }^{*}$ Research supported by NSF-INRIA grant number NSF-Int-0226961.

† Supported by NSF grant number DMS-0408565.

Please use the following format when citing this chapter:

Cagnol, J., Lebiedzik, C.G., and Marchand, R.J., 2006, in IFIP International Federation for Information Processing, Volume 202, Systems, Control, Modeling and Optimization, eds. Ceragioli, F., Dontchev, A., Furuta, H., Marti, K., Pandolfi, L., (Boston: Springer), pp. 67-78. 


\subsection{The Model}

The model to be considered is an extension of Koiter's nonlinear model for a thin shallow shell considered in various forms in $[7,4-1,6,5]$. The shallowness assumption as given in [7] is represented by the expression $L / R \ll 1$ where $R$ is the minimum principal radius of curvature, and $L$ is the wave length of the deformation pattern on the middle surface. We will utilize much of the notation used in [4] and principally developed in [7]. Throughout the paper, we will assume that Greek letters belong to the set $\{1,2\}$ and Latin letters belong to the set $\{1,2,3\}$. We define the middle surface of the shell $S$ as the image of a connected bounded open set $\Omega \subset \mathcal{E}^{2}$ with boundary $\Gamma$ under the mapping $\Phi:\left(\xi^{1}, \xi^{2}\right) \in \bar{\Omega} \rightarrow \mathcal{E}^{3}$ where $\Phi \in\left[C^{3}(\bar{\Omega})\right]^{3}$ and $\mathcal{E}^{n}$ is the ndimensional Euclidean Space. It is assumed that the two tangent vectors given by $\mathbf{a}_{\alpha}=\partial \Phi / \partial \xi^{\alpha}$ are linearly independent at any point on the surface of the shell, and combining these two vectors with the normal vector, $\mathbf{a}_{3}=\frac{\mathbf{a}_{1} \times \mathbf{a}_{2}}{\left|\mathbf{a}_{1} \times \mathbf{a}_{2}\right|}$ defines a covariant basis for a local reference frame on the surface of the shell. We will denote partial derivatives with the notation $\Phi,_{\alpha}=\partial \Phi / \partial \xi^{\alpha}$ for any point $\left(\xi^{1}, \xi^{2}\right) \in \bar{\Omega}$.

In order to simplify the notation, the summation convention will be used in which letters repeated in the roof and cellar of a product will be summed over. For example, the contravariant basis for the tangent plane at any point on the surface of the shell is given by the two vectors $\mathbf{a}^{\beta}$ defined by the relation $\mathbf{a}_{\alpha} \cdot \mathbf{a}^{\beta}=\delta_{\alpha}^{\beta}$ where $\delta_{\alpha}^{\beta}$ denotes the usual Kronecker delta symbol. The matrix $a_{\alpha \beta}=\mathbf{a}^{\alpha} \cdot \mathbf{a}^{\beta}$ is the so-called first fundamental form of the surface with its inverse given by the matrix $\left(a^{\alpha \beta}\right)$. The second fundamental form, denoted by $\left(b_{\alpha \beta}\right)$ measures the normal curvatures of the middle surface of the shell. It is defined by $b_{\alpha \beta}=b_{\beta \alpha}=-\mathbf{a}_{\alpha} \cdot \mathbf{a}_{3, \beta}=\mathbf{a}_{3} \cdot \mathbf{a}_{\alpha, \beta}$.

We represent as $\mathbf{u}=\left(u_{1}, u_{2}, u_{3}\right)$ the vector function of all three displacements, while in-surface displacements are given by $\vec{u}=\left(u_{1}, u_{2}\right)$ and the transverse displacement is denoted by $u_{3}$. The Christoffel symbols given by $\Gamma_{\beta \lambda}^{\alpha}=\mathbf{a}^{\alpha} \cdot \mathbf{a}_{\beta, \lambda}$ are used to define the covariant derivatives for the displacement vector of the middle surface $\mathbf{u}\left(\xi^{1}, \xi^{2}\right)=u_{i} \mathbf{a}^{i}$ in a fixed reference frame. In particular, $u_{\alpha \mid \beta}=u_{\alpha, \beta}-\Gamma_{\alpha \beta}^{\lambda} u_{\lambda}$ and $u_{3 \mid \alpha \beta}=u_{3, \alpha \beta}-\Gamma_{\alpha \beta}^{\lambda} u_{3, \lambda}$. In addition, the linear strain tensor is denoted by $\epsilon_{\alpha \beta}(\vec{u})=\frac{1}{2}\left(u_{\alpha \mid \beta}+u_{\beta \mid \alpha}\right)$. The model assumes that the tangential (in surface) displacements are small relative to the transverse displacement. It is also assumed that all deflections are small and finite in the sense that for any parameter $-1 \leq t \leq 1$ the displacements $t \mathbf{u}$ shall be less than or equal to the middle surface strains in order of magnitude as described in [7]. In this case, suitable expressions for the middle surface strain tensor $\gamma_{\alpha \beta}$ and the change of curvature tensor $\rho_{\alpha \beta}$ are given respectively by $\gamma_{\alpha \beta}(\mathbf{u})=\epsilon_{\alpha \beta}(\vec{u})-b_{\alpha \beta} u_{3}+\frac{1}{2} u_{3, \alpha} u_{3, \beta}$ and $\rho_{\alpha \beta}(\mathbf{u})=u_{3 \mid \alpha \beta}$. We note that the nonlinearity of the model arises from the third term in $\gamma_{\alpha \beta}$. 
These strain measures, along with an appropriate constituitve law, are used to derive corresponding stress measures. The exact form of the stress measures depends on the shell material. Thus, we assume the simplest possible scenario, as done originally by Koiter in [7] and duplicated in [4] and [1]: that the shell consists of elastic, homogeneous, and isotropic material and the strains are small everywhere. Additionally, we assume that all nonzero stress components are imposed on surfaces which are parallel to the middle surface of the shell. Then the tensor of elastic moduli is given by

$$
E^{\alpha \beta \lambda \mu}=\frac{E}{2(1+\nu)}\left[a^{\alpha \lambda} a^{\beta \mu}+a^{\alpha \mu} a^{\beta \lambda}+\frac{2 \nu}{1-\nu} a^{\alpha \beta} a^{\lambda \mu}\right] .
$$

Here, $E$ is Young's modulus and $\nu$ is Poisson's ratio for the material. See [2] for a proof of the positivity of the tensor of elastic moduli. Throughout the exposition, we let $\dot{u}$ represent differentiation with respect to time.

Since our primary interest is weak solutions, we state the model in the following variational form: we look for solutions $u_{\alpha}(t) \in\left[H_{0}^{1}(\Omega)\right]^{2}, u_{3}(t) \in H_{0}^{2}(\Omega)$, $\dot{u}_{\alpha}(t) \in\left[L^{2}(\Omega)\right]^{2}, \dot{u}_{3}(t) \in H_{0}^{1}(\Omega)$ satisfying clamped boundary conditions, $\left.\mathbf{u}\right|_{\Gamma}=\left.\frac{\partial u_{3}}{\partial \nu}\right|_{\Gamma}=0$, such that for test functions $\mathbf{v}=\left(v_{1}, v_{2}, v_{3}\right)=\left(\vec{v}, v_{3}\right) \epsilon$ $\left[H_{0}^{1}(\Omega)\right]^{2} \cap H_{0}^{2}(\Omega)$ we have

$$
m(\ddot{\mathbf{u}}, \mathbf{v})+a(\mathbf{u}, \mathbf{v})-n(\mathbf{u}, \mathbf{v})=0
$$

where

$$
\begin{aligned}
m(\ddot{\mathbf{u}}, v) & =\operatorname{ee}\left\{\left(a^{\alpha \beta} \ddot{u}_{\alpha}, v_{\beta}\right)+\gamma\left(a^{\alpha \beta}\left[b_{1}^{1} b_{2}^{2}-b_{1}^{2} b_{2}^{1}\right] \ddot{u}_{\alpha}, v_{\beta}\right)\right\} \\
& +\operatorname{\rho e\gamma }\left\{\left(a^{\alpha \beta}\left[\ddot{u}_{3 \mid \alpha}+b_{\alpha}^{\lambda} \ddot{u}_{\lambda}\right],\left[v_{3 \mid \beta}+b_{\beta}^{\mu} v_{\mu}\right]\right)\right\} \\
& +\operatorname{ee\gamma }\left\{\left(a^{\alpha \beta} \ddot{u}_{\alpha}, v_{3 \mid \beta} b_{\eta}^{\eta}\right)+\left(a^{\alpha \beta}\left[\ddot{u}_{3 \mid \alpha}+2 b_{\alpha}^{\lambda} \ddot{u}_{\lambda}\right], v_{\beta} b_{\eta}^{\eta}\right)\right\} \\
& +\operatorname{\rho e}\left\{\left(\ddot{u}_{3}, v_{3}\right)+\gamma\left(\left[b_{1}^{1} b_{2}^{2}-b_{1}^{2} b_{2}^{1}\right] \ddot{u}_{3}, v_{3}\right)\right\} \\
a(\mathbf{u}, \mathbf{v})= & e\left(E^{\alpha \beta \lambda \mu}\left[\epsilon_{\alpha \beta}(\vec{u})-b_{\alpha \beta} u_{3}\right], \epsilon_{\lambda \mu}(\vec{v})\right) \\
+ & e \gamma\left(E^{\alpha \beta \lambda \mu} u_{3 \mid \alpha \beta}, v_{3 \mid \lambda \mu}\right)-e\left(E^{\alpha \beta \lambda \mu}\left[\epsilon_{\alpha \beta}(\vec{u})-b_{\alpha \beta} u_{3}\right], b_{\lambda \mu} v_{3}\right) \\
n(\mathbf{u}, \mathbf{v})= & \frac{e}{2}\left(E^{\alpha \beta \lambda \mu} u_{3, \alpha} u_{3, \beta}, \epsilon_{\lambda \mu}(\vec{v})\right)-\frac{e}{2}\left(E^{\alpha \beta \lambda \mu}\left[u_{3, \alpha} u_{3, \beta}\right], b_{\lambda \mu} v_{3}\right) \\
+ & e\left(E^{\alpha \beta \lambda \mu}\left[\epsilon_{\alpha \beta}(\vec{u})-b_{\alpha \beta} u_{3}+\frac{1}{2} u_{3, \alpha} u_{3, \beta}\right], u_{3, \lambda} v_{3, \mu}\right)
\end{aligned}
$$

where $\gamma=e^{2} / 12$ and initial conditions $u_{\alpha}(0)=u_{\alpha}^{0} \in\left[H_{0}^{1}(\Omega)\right]^{2}, \dot{u}_{\alpha}(0)=$ $u_{\alpha}^{1} \in\left[L^{2}(\Omega)\right]^{2}, u_{3}(0)=u_{3}^{0} \in H_{0}^{2}(\Omega), \dot{u}_{3}(0)=u_{3}^{1} \in H_{0}^{1}(\Omega)$ Here we 
make use of the notation $(u, v)=\int_{\Omega} u v \sqrt{a} d \xi_{1} d \xi_{2}$ with $a=\operatorname{det}\left(a_{\alpha \beta}\right)$ and $a \neq 0$. A linear version of this model is considered in [3] to model turbine blades. An associated static (nonlinear) model is given in [4] as an extension of Koiter's model. It accounts for tangential surface loads in order to obtain, as a special case, the nonlinear model for thin elastic plates when there is no curvature, $b_{\alpha \beta}=0$. The energy of the system (1) is $E(t)=E_{p}(t)+E_{k}(t)$ where $E_{k}(t)=m(\dot{\mathbf{u}}, \dot{\mathbf{u}})$ and $E_{p}(t)=a(\mathbf{u}, \mathbf{u})$. It is well-known that $E_{p}(t)$ is topologically equivalent to $\left[H_{0}^{1}(\Omega)\right]^{2} \times H^{2}(\Omega)$.

\section{Main Results}

Lemma 1 (Regular Solutions) For arbitrary $T>0$ and initial data $\left(\vec{u}^{0}, \vec{u}^{1}\right)$ in $\left[H_{0}^{2}(\Omega)\right]^{2} \times\left[H_{0}^{1}(\Omega)\right]^{2},\left(u_{3}^{0}, u_{3}^{1}\right) \in H_{0}^{3}(\Omega) \times H_{0}^{2}(\Omega)$, there exists a unique, global solution to the variational form (1)

$$
\mathbf{u} \in C\left([0, T],\left[H_{0}^{2}(\Omega)\right]^{2} \times H_{0}^{3}(\Omega)\right) \cap C^{1}\left([0, T],\left[H_{0}^{1}(\Omega)\right]^{2} \times H_{0}^{2}(\Omega)\right) .
$$

Proof: The proof of this lemma follows by means of a rather standard nonlinear Galerkin argument. As such, we omit the details.

The existence of finite energy (weak) solutions follows similarly from a standard nonlinear Galerkin argument. However, to our knowledge, uniqueness has until now been an open problem.

Theorem 2 (Weak Solutions) For arbitrary $T>0$ and initial data

$$
\left(\vec{u}^{0}, \vec{u}^{1}\right) \in\left[H_{0}^{1}(\Omega)\right]^{2} \times\left[L^{2}(\Omega)\right]^{2}, \quad\left(u_{3}^{0}, u_{3}^{1}\right) \in H_{0}^{2}(\Omega) \times H_{0}^{1}(\Omega),
$$

there exists a unique solution to the variational form (1) such that

$$
\begin{aligned}
& \left(\vec{u}, u_{3}\right) \in C_{w}\left([0, T] ;\left[H_{0}^{1}(\Omega)\right]^{2} \times H_{0}^{2}(\Omega)\right) \\
& \left(\vec{u}, \dot{u}_{3}\right) \in C_{w}\left([0, T] ;\left[L^{2}(\Omega)\right]^{2} \times H_{0}^{1}(\Omega)\right)
\end{aligned}
$$

The primary mathematical difficulty is due to a lack of regularity in the nonlinear terms. Indeed, the nonlinear terms are not bounded in the space of finite energy. The proof is an adaptation of the method used by Sedenko in [10] and by Lasiecka in [8]. One of the key estimates used in the proof below was proved in [8] and is expressed as the following Lemma.

Lemma 3 Given $\epsilon, r>0$ and $\mathrm{w}^{1} \in\left[H^{\epsilon+r}\right]^{3}$ and $\mathrm{w}^{2} \in\left[H^{1}\right]^{3}$ then

$$
\begin{aligned}
\left\|\left(w_{i}^{1}, w_{i}^{2}\right)\right\|_{L^{2}}^{2} & \leq C\left[\ln \left(1+\lambda_{n}\right)\left\|w_{i}^{1}\right\|_{L^{2}}^{2}+\frac{1}{\lambda_{n}^{r}}\left\|w_{i}^{1}\right\|_{H^{\epsilon+r}}^{2}\right]\left\|w_{i}^{2}\right\|_{H^{1}}^{2} \\
\left\|w_{i}^{1} w_{j}^{2}\right\|_{L^{2}}^{2} & \leq C\left[\ln \left(1+\lambda_{n}\right)\left\|w_{i}^{1}\right\|_{L^{2}}^{2}+\frac{1}{\lambda_{n}^{r}}\left\|w_{i}^{1}\right\|_{H^{\epsilon+r}}^{2}\right]\left\|w_{j}^{2}\right\|_{H^{1}}^{2}
\end{aligned}
$$


where $\lambda_{n}$ denotes the eigenvalues of the Laplace operator. Note the sequence $\left\{\lambda_{n}\right\}$ is increasing with $\lim \lambda_{n}=\infty$.

\section{Proof of Theorem 2}

Let $\tilde{\mathbf{u}}=\mathbf{u}^{2}-\mathbf{u}^{1}$ where $\mathbf{u}^{1}$ and $\mathbf{u}^{2}$ are any two weak solutions of (1) satisfying the same initial conditions. The first step is to derive an abstract formulation for the model. To this end, we make the following definitions.

Definition 4 Define linear operators $\mathcal{M}$ and $\mathcal{A}$ and space $\mathcal{H}$ as

$$
\begin{gathered}
(\mathcal{M} \mathbf{u}, \tilde{\mathbf{u}})=m(\mathbf{u}, \tilde{\mathbf{u}}) ;(\mathcal{A} \mathbf{u}, \tilde{\mathbf{u}})=a(\mathbf{u}, \tilde{\mathbf{u}}) \\
\mathcal{H}=\mathcal{D}\left(\mathcal{A}^{1 / 2}\right) \times \mathcal{D}\left(\mathcal{M}^{1 / 2}\right)=\left[H_{0}^{1}(\Omega)\right]^{2} \times H_{0}^{2}(\Omega) \times\left[\left(L^{2}(\Omega)\right]^{2} \times H_{0}^{1}(\Omega)\right.
\end{gathered}
$$

and let $\mathbf{A}: \mathcal{H} \rightarrow \mathcal{H}$ be defined by

$$
\mathbf{A}=\left(\begin{array}{cc}
0 & -I \\
\mathcal{M}^{-1} \mathcal{A} & 0
\end{array}\right)
$$

The coercivity of $m(\mathbf{u}, \tilde{\mathbf{u}})$ and $a(\mathbf{u}, \tilde{\mathbf{u}})$ were proved in [3] and [4] respectively, so the operator $\mathbf{A}$ is well-defined. Also, if we define the nonlinear operator $N$ such that $(N(\mathbf{u}), \mathbf{v})=n(\mathbf{u}, \mathbf{v})$ with $N(\mathbf{u})=N_{\alpha}\left(u_{3}\right)+N_{3}\left(\vec{u}, u_{3}\right)$ where

$$
\begin{aligned}
N_{\alpha}\left(u_{3}\right)= & \left.\frac{e}{2}\left(E^{\alpha \beta \lambda \mu} u_{3, \lambda}, u_{3, \mu}\right)\right|_{\beta} \\
N_{3}\left(\vec{u}, u_{3}\right)= & -\frac{e}{2}\left(b_{\alpha \beta} E^{\alpha \beta \lambda \mu} u_{3, \lambda} u_{3, \mu}\right) \\
& \quad-\left.e\left(u_{3 \mid \alpha} E^{\alpha \beta \lambda \mu}\left[\epsilon_{\lambda \mu}(\vec{u})-b_{\lambda \mu} u_{3}+\frac{1}{2} u_{3, \lambda} u_{3, \mu}\right]\right)\right|_{\beta}
\end{aligned}
$$

then the variational form in (1) implies

$$
\partial_{t}\left(\begin{array}{c}
\mathbf{u} \\
\partial_{t} \mathbf{u}
\end{array}\right)+\mathbf{A}\left(\begin{array}{c}
\mathbf{u} \\
\partial_{t} \mathbf{u}
\end{array}\right)=\left(\begin{array}{c}
0 \\
\mathcal{M}^{-1} N(\mathbf{u})
\end{array}\right)
$$

This will be used to prove the following lemma.

LEMMA 5 Using Definition 4 and (7), the following holds.

$$
\left\|\mathbf{A}^{-1}\left(\begin{array}{c}
\tilde{\mathbf{u}} \\
\partial_{t} \tilde{\mathbf{u}}
\end{array}\right)\right\|_{\mathcal{H}} \leq \int_{0}^{t}\left\|\mathbf{A}^{-1}\left(\begin{array}{c}
0 \\
\mathcal{M}^{-1}\left[N\left(\mathbf{u}^{2}\right)-N\left(\mathbf{u}^{1}\right)\right]
\end{array}\right)\right\|_{\mathcal{H}} d s
$$


Proof: Acting with $\mathbf{A}^{-1}$ on equation (7) and multiplying by $X(s)$ yields

$$
\begin{gathered}
\left(\partial_{t} \mathbf{A}^{-1}\left(\begin{array}{c}
\tilde{\mathbf{u}} \\
\partial_{t} \tilde{\mathbf{u}}
\end{array}\right), X(s)\right)_{\mathcal{H}}+\left(\mathbf{A A}^{-1}\left(\begin{array}{c}
\tilde{\mathbf{u}} \\
\partial_{t} \tilde{\mathbf{u}}
\end{array}\right), X(s)\right)_{\mathcal{H}} \\
=\left(\mathbf{A}^{-1}\left(\begin{array}{c}
0 \\
\mathcal{M}_{\gamma}^{-1}\left[N\left(\mathbf{u}^{2}\right)-N\left(\mathbf{u}^{1}\right)\right]
\end{array}\right), X(s)\right)_{\mathcal{H}}
\end{gathered}
$$

We define

$$
X(s)=\mathbf{A}^{-1}\left(\begin{array}{c}
\tilde{\mathbf{u}}(s) \\
\partial_{t} \tilde{\mathbf{u}}(s)
\end{array}\right) \text { and } F(s)=\mathbf{A}^{-1}\left(\begin{array}{c}
0 \\
\mathcal{M}^{-1}\left[N\left(\mathbf{u}^{2}\right)-N\left(\mathbf{u}^{1}\right)\right]
\end{array}\right)
$$

Using $(\mathbf{A} X, X)_{\mathcal{H}}=0$ on (9) yields $\left(\partial_{t} X(s), X(s)\right)_{\mathcal{H}}=(X(s), F(s))_{\mathcal{H}}$ and

$$
\frac{1}{2} \int_{0}^{t} \partial_{s}\|X(s)\|_{\mathcal{H}}^{2} d s=\int_{0}^{t}(X(s), F(s))_{\mathcal{H}} d s
$$

it follows

$$
\frac{1}{2}\|X(t)\|_{\mathcal{H}}^{2} \leq \frac{1}{2}\|X(0)\|_{\mathcal{H}}^{2}+\int_{0}^{t}\|X(s)\|_{\mathcal{H}}\|F(s)\|_{\mathcal{H}} d s
$$

Since $\tilde{\mathbf{u}}(0)=\partial_{t} \tilde{\mathbf{u}}(0)=0$ and $\mathbf{A}^{-1}$ is linear, we have $X(0)=0$, so

$$
\frac{1}{2}\|X(t)\|_{\mathcal{H}}^{2} \leq\left(\sup _{s \in[0, t]}\|X(s)\|_{\mathcal{H}}\right)\left(\int_{0}^{t}\|F(s)\|_{\mathcal{H}} d s\right)
$$

thus, for all $\bar{t} \leq t$ we have

$$
\left\{\begin{array}{l}
\frac{1}{2}\|X(\bar{t})\|_{\mathcal{H}}^{2} \leq\left(\sup _{s \in[0, \bar{t}]}\|X(s)\|_{\mathcal{H}}\right)\left(\int_{0}^{\bar{t}}\|F(s)\|_{\mathcal{H}} d s\right) \\
\sup _{s \in[0, \bar{t} t}\|X(s)\|_{\mathcal{H}}^{2} \leq \sup _{s \in[0, t]}\|X(s)\|_{\mathcal{H}}^{2} \\
\int_{0}^{\bar{t}}\|F(s)\|_{\mathcal{H}} d s \leq \int_{0}^{t}\|F(s)\|_{\mathcal{H}} d s
\end{array}\right.
$$

Combining (10) and (11) gives

$$
\frac{1}{2} \sup _{\bar{t} \in[0, t]}\|X(\bar{t})\|_{\mathcal{H}}^{2} \leq\left(\sup _{s \in[0, t]}\|X(s)\|_{\mathcal{H}}^{2}\right)\left(\int_{0}^{t}\|F(s)\|_{\mathcal{H}} d s\right)
$$

consequently

$$
\sup _{s \in[0, t]}\|X(s)\|_{\mathcal{H}} \leq \int_{0}^{t}\|F(s)\|_{\mathcal{H}} d s
$$

and then using $\|X(t)\|_{\mathcal{H}} \leq \sup _{s \in[0, t]}\|X(s)\|_{\mathcal{H}}$, we get (8). 
LEMMA 6 The following inequality holds.

$$
\left\|\mathcal{M}^{1 / 2} \tilde{\mathbf{u}}\right\|_{L^{2}} \leq\left\|\mathbf{A}^{-1}\left(\begin{array}{c}
\tilde{\mathbf{u}} \\
\partial_{t} \tilde{\mathbf{u}}
\end{array}\right)\right\|_{\mathcal{H}}
$$

Proof: We have

$$
\mathbf{A}^{-1}\left(\begin{array}{c}
\tilde{\mathbf{u}} \\
\partial_{t} \tilde{\mathbf{u}}
\end{array}\right)=\left(\begin{array}{c}
\mathcal{A}^{-1} \mathcal{M} \partial_{t} \tilde{\mathbf{u}} \\
-\tilde{\mathbf{u}}
\end{array}\right)
$$

therefore

$$
\left\|\mathbf{A}^{-1}\left(\begin{array}{c}
\tilde{\mathbf{u}} \\
\partial_{t} \tilde{\mathbf{u}}
\end{array}\right)\right\|_{\mathcal{H}}^{2}=\left\|\mathcal{A}^{-1 / 2} \mathcal{M} \partial_{t} \tilde{\mathbf{u}}\right\|_{L^{2}}^{2}+\left\|\mathcal{M}^{1 / 2} \tilde{\mathbf{u}}\right\|_{L^{2}}^{2}
$$

LEMMA 7 The following inequality holds.

$$
\left\|\mathcal{M}^{1 / 2} \tilde{\mathbf{u}}\right\|_{L^{2}} \leq \int_{0}^{t}\left\|\mathcal{A}^{-1 / 2}\left[N\left(\mathbf{u}^{2}(s)\right)-N\left(\mathbf{u}^{1}\right)(s)\right]\right\|_{L^{2}} d s
$$

Proof: We have

$$
\mathbf{A}^{-1}\left(\begin{array}{c}
0 \\
\mathcal{M}^{-1}\left[N\left(\mathbf{u}^{2}\right)-N\left(\mathbf{u}^{1}\right)\right]
\end{array}\right)=\left(\begin{array}{c}
\mathcal{A}^{-1}\left[N\left(\mathbf{u}^{2}\right)-N\left(\mathbf{u}^{1}\right)\right] \\
0
\end{array}\right)
$$

therefore

$$
\left\|\mathbf{A}^{-1}\left(\begin{array}{c}
0 \\
\mathcal{M}^{-1}\left[N\left(\mathbf{u}^{2}\right)-N\left(\mathbf{u}^{1}\right)\right]
\end{array}\right)\right\|_{\mathcal{H}}=\left\|\mathcal{A}^{-1 / 2}\left[N\left(\mathbf{u}^{2}\right)-N\left(\mathbf{u}^{1}\right)\right]\right\|_{L^{2}}
$$

From Lemma 5, we get

$$
\left\|\mathbf{A}^{-1}\left(\begin{array}{c}
\tilde{\mathbf{u}} \\
\partial_{t} \tilde{\mathbf{u}}
\end{array}\right)\right\|_{\mathcal{H}} \leq \int_{0}^{t}\left\|\mathcal{A}^{-1 / 2}\left[N\left(\mathbf{u}^{2}\right)-N\left(\mathbf{u}^{\mathbf{1}}\right)\right]\right\|_{L^{2}} d s
$$

Applying Lemma 6 yields (13).

LEMMA 8 Following Lemma 7, we obtain

$$
\begin{aligned}
\left\|\tilde{u}_{\alpha}\right\|_{L^{2}}^{2}+\left\|\tilde{u_{3}}\right\|_{H^{1}}^{2} \leq t \int_{0}^{t}\left(\| N_{\alpha}\left(u_{3}^{2}\right)\right. & -N_{\alpha}\left(u^{1}\right) \|_{H^{-1}}^{2} \\
& \left.+\left\|N_{3}\left(\vec{u}^{2}, u_{3}^{2}\right)-N_{3}\left(\vec{u}^{1}, u_{3}^{1}\right)\right\|_{H^{-2}}^{2}\right) d s
\end{aligned}
$$

Proof: The lemma follows from the use of $\left(\int_{0}^{t} f(t) d t\right)^{2} \leq t \int_{0}^{t} f(t)^{2} d t$ on (13). 
LeMma 9 Given $\epsilon>0$ and $r>0$ with $\epsilon+r \leq 1$, there exists a constant $C$ depending on $\lambda, n, u_{3}^{1}$, and $u_{3}^{2}$ such that

$$
\left\|N_{\alpha}\left(u_{3}^{2}\right)-N_{\alpha}\left(u_{3}^{1}\right)\right\|_{H^{-1}} \leq C \ln \left(1+\lambda_{n}\right)\left\|\tilde{u}_{3}\right\|_{H^{1}}+\frac{C}{\lambda_{n}^{r}}
$$

Proof: The proof uses the positivity of the tensor of elastic moduli given in [2] and the fact that the divergence operator, $\left.(\cdot)\right|_{\beta}$, is a bounded operator on $H^{-1}(\Omega)$. Recalling the definition of $N_{\alpha}\left(u_{3}\right)$, it can be shown that

$$
\begin{aligned}
N_{\alpha}\left(u_{3}^{2}\right)-N_{\alpha}\left(u_{3}^{1}\right) & =\left.\frac{e}{2}\left[E^{\alpha \beta \lambda \mu}\left(u_{3, \lambda}^{2} u_{3, \mu}^{2}-u_{3, \lambda}^{1} u_{3, \mu}^{1}\right)\right]\right|_{\beta} \\
& =\left.\frac{e}{2}\left[E^{\alpha \beta \lambda \mu} \tilde{u}_{3, \lambda}\left(u_{3, \mu}^{2}+u_{3, \mu}^{1}\right)\right]\right|_{\beta}
\end{aligned}
$$

This gives

$$
\begin{aligned}
\left\|N_{\alpha}\left(u_{3}^{2}\right)-N_{\alpha}\left(u_{3}^{1}\right)\right\|_{H^{-1}} & =\left\|\left.\frac{e}{2}\left[E^{\alpha \beta \lambda \mu} \tilde{u}_{3, \lambda}\left(u_{3, \mu}^{2}+u_{3, \mu}^{1}\right)\right]\right|_{\beta}\right\|_{H^{-1}} \\
& \leq C\left\|\tilde{u}_{3, \lambda}\left(u_{3, \mu}^{2}+u_{3, \mu}^{1}\right)\right\|_{L^{2}}
\end{aligned}
$$

Now apply Lemma 3 to obtain

$$
\left\|\tilde{u}_{3, \lambda} u_{3, \lambda}^{2}\right\| \leq C\left(\ln \left(1+\lambda_{n}\right)\left\|\tilde{u}_{3}\right\|_{H^{1}}+\frac{1}{\lambda_{n}^{r}}\left\|u_{3}^{2}-u_{3}^{1}\right\|_{H^{1+\epsilon+r}}\right)\left\|u_{3}^{2}\right\|_{H^{2}}
$$

Combining this result with an analogous result for $\left\|\tilde{u}_{3, \lambda} u_{3, \lambda}^{2}\right\|$ and (15) and using the fact that $u_{3}^{1}$ amd $u_{3}^{2}$ are $H^{2}$ functions leads to the conclusion of the lemma. We now prove a similar lemma for $N_{3}\left(\vec{u}, u_{3}\right)$.

LEMMA 10 Given $\epsilon>0$ and $r>0$ with $\epsilon+r \leq 1$, there exists a constant $C$ depending on $\lambda, n, \mathbf{u}^{1}$, and $\mathbf{u}_{3}^{2}$ such that

$$
\begin{aligned}
& \left\|N_{3}\left(\vec{u}^{2}, u_{3}^{2}\right)-N_{3}\left(\vec{u}^{1}, u_{3}^{1}\right)\right\|_{H^{-2}} \\
& \quad \leq C \ln \left(1+\lambda_{n}\right)\left\|\tilde{u}_{3}\right\|_{H^{1}}^{2}+C \ln \left(1+\lambda_{n}\right)\left\|\tilde{u}_{\alpha}\right\|_{L^{2}}^{2}+\frac{C}{\lambda_{n}^{r}}
\end{aligned}
$$

Proof. Using the definition of $N_{3}\left(\vec{u}, u_{3}\right)$ it can be shown that

$$
N_{3}\left(u_{\alpha}^{2}, u_{3}^{2}\right)-N_{3}\left(u_{\alpha}^{1}, u_{3}^{1}\right)=X^{1}\left(\tilde{u}_{3}, u_{3}^{1}, u_{3}^{2}\right)+X^{2}\left(\tilde{\mathbf{u}}, \mathbf{u}^{1}, \mathbf{u}^{2}\right)
$$


with

$$
\begin{aligned}
& X^{1}\left(\tilde{u}_{3}, u_{3}^{1}, u_{3}^{2}\right)=-\frac{e}{2} b_{\alpha \beta} E^{\alpha \beta \lambda \mu} \tilde{u}_{3, \lambda}\left(u_{3, \mu}^{2}+u_{3, \mu}^{1}\right) \\
& \quad+\left.e\left(u_{3 \mid \alpha}^{2} E^{\alpha \beta \lambda \mu} b_{\lambda \mu} \tilde{u}_{3}+\tilde{u}_{3 \mid \alpha} E^{\alpha \beta \lambda \mu} b_{\lambda \mu} u_{3}^{1}\right)\right|_{\beta} \\
& \quad-\left.\frac{e}{2}\left(u_{3 \mid \alpha}^{2} E^{\alpha \beta \lambda \mu} u_{3, \lambda}^{2} \tilde{u}_{3, \mu}+u_{3 \mid \alpha}^{2} E^{\alpha \beta \lambda \mu} \tilde{u}_{3, \lambda} u_{3, \mu}^{1}+\tilde{u}_{3 \mid \alpha} E^{\alpha \beta \lambda \mu} u_{3, \lambda}^{1} u_{3, \mu}^{1}\right)\right|_{\beta} \\
& X^{2}\left(\tilde{\mathbf{u}}, \mathbf{u}^{1}, \mathbf{u}^{2}\right)=\left.e\left(u_{3 \mid \alpha}^{2} E^{\alpha \beta \lambda \mu} \epsilon_{\lambda \mu}(\vec{u})+\tilde{u}_{3 \mid \alpha} E^{\alpha \beta \lambda \mu} \epsilon_{\lambda \mu}\left(\vec{u}^{1}\right)\right)\right|_{\beta}
\end{aligned}
$$

Consequently,

$$
\begin{aligned}
\| N_{3}\left(\vec{u}^{2}, u_{3}^{2}\right) & -N_{3}\left(\vec{u}^{1}, u_{3}^{1}\right) \|_{H^{-2}} \\
& \leq\left\|X^{1}\left(\tilde{u}_{3}, u_{3}^{1}, u_{3}^{2}\right)\right\|_{H^{-2}}+\left\|X^{2}\left(\tilde{\mathbf{u}}, \mathbf{u}^{1}, \mathbf{u}^{2}\right)\right\|_{H^{-2}}
\end{aligned}
$$

It suffices to estimate each term on the right-hand side of (16). In particular,

$$
\begin{aligned}
& \left\|X^{1}\left(\tilde{u}_{3}, u_{3}^{1}, u_{3}^{2}\right)\right\|_{H^{-2}} \leq C\left\{\left\|b_{\alpha \beta} E^{\alpha \beta \lambda \mu} \tilde{u}_{3, \lambda}\left(u_{3, \mu}^{2}+u_{3, \mu}^{1}\right)\right\|_{H^{-2}}\right. \\
& +\left\|u_{3 \mid \alpha}^{2} E^{\alpha \beta \lambda \mu} b_{\lambda \mu} \tilde{u}_{3}+\tilde{u}_{3 \mid \alpha} E^{\alpha \beta \lambda \mu} b_{\lambda \mu} u_{3}^{1}\right\|_{H^{-1}} \\
& \left.-\left\|u_{3 \mid \alpha}^{2} E^{\alpha \beta \lambda \mu} u_{3, \lambda}^{2} \tilde{u}_{3, \mu}+u_{3 \mid \alpha}^{2} E^{\alpha \beta \lambda \mu} \tilde{u}_{3, \lambda} u_{3, \mu}^{1}+\tilde{u}_{3 \mid \alpha} E^{\alpha \beta \lambda \mu} u_{3, \lambda}^{1} u_{3, \mu}^{1}\right\|_{H^{-1}}\right\},
\end{aligned}
$$

Using classical embedding theory and the fact that the second fundamental form and the tensor of elastic moduli are bounded yields the following.

$$
\begin{aligned}
& \left\|b_{\alpha \beta} E^{\alpha \beta \lambda \mu} \tilde{u}_{3, \lambda}\left(u_{3, \mu}^{2}+u_{3, \mu}^{1}\right)\right\|_{H^{-2}} \leq C\left\|\tilde{u}_{3, \lambda}\left(u_{3, \mu}^{2}+u_{3, \mu}^{1}\right)\right\|_{L^{2}} \\
\leq & C\left\|\tilde{u}_{3}\right\|_{H^{1}}\left\|u_{3}^{2}+u_{3}^{1}\right\|_{H^{1}} \leq C\left\|\tilde{u}_{3}\right\|_{H^{1}}
\end{aligned}
$$

Applying similar arguments to the remaining terms in (17) leads to

$$
\left\|X^{1}\left(\tilde{u}_{3}, u_{3}^{1}, u_{3}^{2}\right)\right\|_{H^{-2}} \leq C\left\|\tilde{u}_{3}\right\|_{H^{1}}
$$

The higher order terms in $X^{2}$ make it much more difficult to estimate. First,

$$
\begin{aligned}
& \left\|X^{2}\left(\tilde{\mathbf{u}}, \mathbf{u}^{1}, \mathbf{u}^{2}\right)\right\|_{H^{-2}} \\
& \quad \leq C\left\|\tilde{u}_{3 \mid \alpha} E^{\alpha \beta \lambda \mu} \epsilon_{\lambda \mu}\left(\vec{u}^{1}\right)\right\|_{H^{-1}}+C\left\|u_{3 \mid \alpha}^{2} E^{\alpha \beta \lambda \mu} \epsilon_{\lambda \mu}(\overrightarrow{\vec{u}})\right\|_{H^{-}}
\end{aligned}
$$

Applying Lemma 3 to the first term in (19) gives

$$
\left\|\tilde{u}_{3 \mid \alpha} E^{\alpha \beta \lambda \mu} \epsilon_{\lambda \mu}\left(\vec{u}^{1}\right)\right\|_{H^{-1}}=\sup _{\left\{\|\varphi\|_{H_{0}^{1}}=1\right\}} \int_{\Omega} \tilde{u}_{3 \mid \alpha} E^{\alpha \beta \lambda \mu} \epsilon_{\lambda \mu}\left(\vec{u}^{1}\right) \varphi_{\beta} \sqrt{a} d \xi^{1} d \xi^{2}
$$




$$
\begin{aligned}
& \leq \sup _{\left\{\varphi,\|\varphi\|_{H_{0}^{1}}=1\right\}} C\left\|\epsilon_{\lambda \mu}\left(\vec{u}^{1}\right)\right\|_{L^{2}}\left\|\tilde{u}_{3 \mid \alpha} \varphi_{\beta}\right\|_{L^{2}} \\
& \leq C\left(\left\|u^{1}\right\|_{H^{1}}\left(\ln \left(1+\lambda_{n}\right)\left\|\tilde{u}_{3}\right\|_{H^{1}}+\frac{1}{\lambda_{n}^{r}}\left\|\tilde{u}_{3}\right\|_{H^{1+\epsilon+r}}\right)\right.
\end{aligned}
$$

after applying the supremum. Similarly,

$$
\begin{aligned}
& \left\|u_{3 \mid \alpha}^{2} E^{\alpha \beta \lambda \mu} \epsilon_{\lambda \mu}(\overrightarrow{\vec{u}})\right\|_{H^{-1}}=\sup _{\left\{\|\varphi\|_{H_{0}^{1}}=1\right\}} \int_{\Omega} E^{\alpha \beta \lambda \mu} u_{3 \mid \alpha}^{2} \varphi_{\beta} \epsilon_{\lambda \mu}(\overrightarrow{\vec{u}}) \sqrt{a} d \xi^{1} d \xi^{2} \\
& =\sup _{\left\{\varphi,\|\varphi\|_{H_{0}^{1}}=1\right\}} \int_{\Omega} E^{\alpha \beta \lambda \mu} u_{3 \mid \alpha}^{2} \varphi_{\beta}\left(\frac{1}{2}\left(\tilde{u}_{\lambda \mid \mu}+\tilde{u}_{\mu \mid \lambda}\right)\right) \sqrt{a} d \xi^{1} d \xi^{2}(21) \\
& =\quad \sup _{\left\{\varphi,\|\varphi\|_{H_{0}^{1}}=1\right\}} \frac{1}{2} \int_{\Omega} E^{\alpha \beta \lambda \mu} u_{3 \mid \alpha}^{2} \varphi_{\beta} \\
& \\
& \quad\left(\tilde{u}_{\lambda, \mu}-\Gamma_{\lambda \mu}^{\delta} \tilde{u}_{\delta}+\tilde{u}_{\mu, \lambda}-\Gamma_{\mu \lambda}^{\delta} \tilde{u}_{\delta}\right) \sqrt{a} d \xi^{1} d \xi^{2}
\end{aligned}
$$

where the definitions of the linear strain $\left(\epsilon_{\lambda \mu}\right)$ and covariant differentiation were used in (21) and (22). Using the clamped boundary conditions,

$$
\int_{\Omega} E^{\alpha \beta \lambda \mu} u_{3 \mid \alpha}^{2} \varphi_{\beta} \tilde{u}_{\lambda, \mu} \sqrt{a} d \xi^{1} d \xi^{2}=-\left.\int_{\Omega} E^{\alpha \beta \lambda \mu}\left(u_{3 \mid \alpha}^{2} \varphi_{\beta}\right)\right|_{\mu} \tilde{u}_{\lambda} \sqrt{a} d \xi^{1} d \xi^{2}
$$

which applied to (22) yields

$$
\begin{gathered}
\left\|u_{3 \mid \alpha}^{2} E^{\alpha \beta \lambda \mu} \epsilon_{\lambda \mu}(\overrightarrow{\vec{u}})\right\|_{H^{-1}} \\
=\sup _{\left\{\varphi,\|\varphi\|_{H_{0}^{1}}=1\right\}} \frac{1}{2}\left\{\int_{\Omega} E^{\alpha \beta \lambda \mu}\left(\left.\left(u_{3 \mid \alpha}^{2} \varphi_{\beta}\right)\right|_{\mu} \tilde{u}_{\lambda}+\left.\left(u_{3 \mid \alpha}^{2} \varphi_{\beta}\right)\right|_{\lambda} \tilde{u}_{\mu}\right) \sqrt{a} d \xi^{1} d \xi^{2}\right. \\
\left.\quad-\int_{\Omega} E^{\alpha \beta \lambda \mu}\left(\Gamma_{\lambda \mu}^{\delta} \tilde{u}_{\delta}+\Gamma_{\mu \lambda}^{\delta} \tilde{u}_{\delta}\right) u_{3 \mid \alpha}^{2} \varphi_{\beta} \sqrt{a} d \xi^{1} d \xi^{2}\right\} \\
\leq \sup _{\left\{\varphi,\|\varphi\|_{H_{0}^{1}}=1\right\}} C\left\|\left.\left(u_{3 \mid \alpha}^{2} \varphi_{\beta}\right)\right|_{\mu} \tilde{u}_{\lambda}\right\|_{L^{2}}+C\left\|\left.\left(u_{3 \mid \alpha}^{2} \varphi_{\beta}\right)\right|_{\lambda} \tilde{u}_{\mu}\right\|_{L^{2}} \\
\left.\quad+C\left\|\Gamma_{\lambda \mu}^{\delta} \tilde{u}_{\delta} u_{3 \mid \alpha}^{2} \varphi_{\beta}\right\|_{L^{2}}+C \| \Gamma_{\mu \lambda}^{\delta} \tilde{u}_{\delta} u_{3 \mid \alpha}^{2} \varphi_{\beta}\right) \|_{L^{2}}
\end{gathered}
$$

Expanding the divergence and using Cauchy-Schwarz gives

$$
\begin{aligned}
\left\|\left.\left(u_{3 \mid \alpha}^{2} \varphi_{\beta}\right)\right|_{\mu} \tilde{u}_{\lambda}\right\|_{L^{2}} \leq\left\|u_{3 \mid \alpha \mu}^{2}\right\|\left\|_{L^{2}}\right\| \varphi_{\beta} \tilde{u}_{\lambda}\left\|_{L^{2}}+\right\| \varphi_{\beta \mid \mu}\left\|_{L^{2}}\right\| u_{3 \mid \alpha}^{2} \tilde{u}_{\lambda} \|_{L^{2}} \\
\leq\left\|u_{3}^{2}\right\|_{H^{2}}\left\|\varphi_{\beta} \tilde{u}_{\lambda}\right\|_{L^{2}}+\left\|\varphi_{\beta}\right\|_{H^{1}}\left\|u_{3 \mid \alpha}^{2} \tilde{u}_{\lambda}\right\|_{L^{2}} \\
\leq C\left\|u_{3}^{2}\right\|_{H^{2}}\left[\ln \left(1+\lambda_{n}\right)\left\|\tilde{u}_{\lambda}\right\|_{L^{2}}+\frac{1}{\lambda_{n}^{r}}\left\|\tilde{u}_{\lambda}\right\|_{H^{r+\epsilon}}\right]\left\|\varphi_{\beta}\right\|_{H^{1}}
\end{aligned}
$$




$$
+C\left\|\varphi_{\beta}\right\|_{H^{1}}\left[\ln \left(1+\lambda_{n}\right)\left\|\tilde{u}_{\lambda}\right\|_{L^{2}}+\frac{1}{\lambda_{n}^{r}}\left\|\tilde{u}_{\lambda}\right\|_{H^{r+\epsilon}}\right]\left\|u_{3}^{2}\right\|_{H^{2}}
$$

for the first term in (24). A similar argument for the third term on the right-hand side of (24) leads to

$$
\left\|\Gamma_{\lambda \mu}^{\delta} \tilde{u}_{\delta} u_{3 \mid \alpha}^{2} \varphi_{\beta}\right\|_{L^{2}} \leq C\left[\ln \left(1+\lambda_{n}\right)\left\|\tilde{u}_{\delta}\right\|_{L^{2}}+\frac{1}{\lambda_{n}^{r}}\left\|\tilde{u}_{\lambda}\right\|_{H^{r+\epsilon}}\right]\left\|\varphi_{\beta}\right\|_{H^{1}}\left\|u_{3}^{2}\right\|_{H^{1}}
$$

Combining these with similar arguments for the remaining terms gives

$$
\left\|u_{3 \mid \alpha}^{2} E^{\alpha \beta \lambda \mu} \epsilon_{\lambda \mu}(\overrightarrow{\vec{u}})\right\|_{H^{-1}} \leq C\left[\ln \left(1+\lambda_{n}\right)\left\|\tilde{u}_{\alpha}\right\|_{L^{2}}+\frac{1}{\lambda_{n}^{r}}\left\|\tilde{u}_{\alpha}\right\|_{H^{r+\epsilon}}\right]\left\|u_{3}^{2}\right\|_{H^{2}} .
$$

Combining (16), (18), (19),(20), and (27) results in Lemma (10).

LEMMA 11 Let $r=1-\epsilon$, then

$$
\left\|\tilde{u}_{\alpha}\right\|_{L^{2}}^{2}+\left\|\tilde{u}_{3}\right\|_{H^{1}}^{2} \leq C t^{2} \lambda_{n}^{C t^{2}-r / 2}\left(1+\lambda_{n}^{-1}\right)^{C t^{2}}
$$

Proof: Lemmas 7, 9, and 10 give

$$
\left\|\tilde{u}_{\alpha}\right\|_{L^{2}}^{2}+\left\|\tilde{u}_{3}\right\|_{H^{1}}^{2} \leq C t \int_{0}^{t}\left[\ln \left(1+\lambda_{n}\right)\left\|\tilde{u}_{3}\right\|_{H^{1}}^{2}+\ln \left(1+\lambda_{n}\right)\left\|\tilde{u}_{\alpha}\right\|_{L^{2}}^{2}+\frac{1}{\lambda_{n}^{r}}\right] d s
$$

which implies

$$
\left\|\tilde{u}_{\alpha}\right\|_{L^{2}}^{2}+\left\|\tilde{u}_{3}\right\|_{H^{1}}^{2} \leq \frac{C t^{2}}{\lambda_{n}^{r / 2}}+C t \ln \left(1+\lambda_{n}\right) \int_{0}^{t}\left(\left\|\tilde{u}_{3}\right\|_{H^{1}}+\left\|\tilde{u}_{\alpha}\right\|_{L^{2}}\right) d s
$$

Applying Gronwall's inequality to (29) gives (28).

Since the sequence $\left\{\lambda_{n}\right\}$ tends to $\infty$, the right-hand side of (28)will go to zero as long as $t \in\left[0, \sqrt{\frac{r}{2 C}}\right)$. Thus, $\tilde{\mathbf{u}}=0$ on $\left[0, \sqrt{\frac{r}{2 C}}\right)$. The bootstrap argument completes the proof of the theorem.

\section{References}

[1] M. Bernadou. Méthodes d'Eléments Finis pour les Problémes de Coques Minces. Masson, Paris, 1994.

[2] M. Bernadou, P.G. Ciarlet and B. Miara. Existence theorems for two-dimensional linear shell theories. Technical Report 1771. Unité deRecherche INRIA-Rocquencourt, 1992.

[3] M. Bernadou, B. Lalanne. On the approximation of free vibration modes of a general thin shell application to turbine blades. In The Third European Conference on Mathematics in Industry. J. Manley, et al. Eds., Kluwer Academic Publishers and B. G. Teubner Stuttgart, 257-264, 1990. 
[4] M. Bernadou and J.T. Oden. An existence theorem for a class of nonlinear shallow shell problems. J. Math Pures Appl. 60:285-308, 1981.

[5] P.G. Ciarlet. A two-dimensional non-linear shell model of Koiter's type. Jean Leray '99 Conference Proceedings. Kluwer Academic Publishers, 437-449, 2003.

[6] M.C. Delfour and J.P. Zolésio. Tangential differential equations for dynamical thin/shallow shells. J. Differential Equations. 128:125-167, 1995.

[7] W.T. Koiter. On the nonlinear theory of thin elastic shells. Proc. Kon. Ned. Akad. Wetensch. B: 1 -54, 1966.

[8] I. Lasiecka. Uniform stabilizability of a full von Karman system with nonlinear boundary feedback. SIAM J. Control Optim. 36: 1376-1422, 1998.

[9] I. Lasiecka, R. Marchand. Riccati equations arising in acoustic structure interactions with curved walls. Journal of Dynamics and Control. 8:269-292, 1998.

[10] V.I. Sedenko. On uniqueness of the generalized solutions of initial boundary value problems for Marguerre-Vlasov nonlinear oscillations of shallow shells. Russian Izv. 1-2, 1994. 